\title{
Monte Carlo simulations of a scintillation camera using GATE: validation and application modelling
}

\author{
Steven Staelens ${ }^{1}$, Daniel Strul ${ }^{2}$, Giovanni Santin ${ }^{3}$, \\ Stefaan Vandenberghe ${ }^{1}$, Michel Koole ${ }^{1}$, Yves D’Asseler ${ }^{1}$, \\ Ignace Lemahieu $^{1}$ and Rik Van de Walle ${ }^{1}$ \\ ${ }^{1}$ ELIS Department, Ghent University Sint-Pietersnieuwstraat, 41 B-9000 Ghent, Belgium \\ 2 IPHE, University of Lausanne, CH-1015 Lausanne, Switzerland \\ ${ }^{3}$ ESA-ESTEC, Keplerlaan 1, PO Box 299 NL-2200, AG Noordwijk, The Netherlands \\ E-mail: Steven.Staelens@ugent.be
}

Received 22 May 2003, in final form 21 July 2003

Published 3 September 2003

Online at stacks.iop.org/PMB/48/3021

\begin{abstract}
Geant4 application for tomographic emission (GATE) is a recently developed simulation platform based on Geant4, specifically designed for PET and SPECT studies. In this paper we present validation results of GATE based on the comparison of simulations against experimental data, acquired with a standard SPECT camera. The most important components of the scintillation camera were modelled. The photoelectric effect, Compton and Rayleigh scatter are included in the gamma transport process. Special attention was paid to the processes involved in the collimator: scatter, penetration and lead fluorescence. A LEHR and a MEGP collimator were modelled as closely as possible to their shape and dimensions. In the validation study, we compared the simulated and measured energy spectra of different isotopes: ${ }^{99 \mathrm{~m}} \mathrm{Tc},{ }^{22} \mathrm{Na},{ }^{57} \mathrm{Co}$ and ${ }^{67} \mathrm{Ga}$. The sensitivity was evaluated by using sources at varying distances from the detector surface. Scatter component analysis was performed in different energy windows at different distances from the detector and for different attenuation geometries. Spatial resolution was evaluated using a ${ }^{99 \mathrm{~m}} \mathrm{Tc}$ source at various distances. Overall results showed very good agreement between the acquisitions and the simulations. The clinical usefulness of GATE depends on its ability to use voxelized datasets. Therefore, a clinical extension was written so that digital patient data can be read in by the simulator as a source distribution or as an attenuating geometry. Following this validation we modelled two additional camera designs: the Beacon transmission device for attenuation correction and the Solstice scanner prototype with a rotating collimator. For the first setup a scatter analysis was performed and for the latter design, the simulated sensitivity results were compared against theoretical predictions. Both case studies demonstrated the flexibility and accuracy of GATE and exemplified its potential benefits in protocol optimization and in system design.
\end{abstract}




\section{Introduction}

Monte Carlo is a numerical calculation method based on the technique of random variable sampling. It has been used in mathematics from the 18th century onwards, but only came into use in nuclear physics since the rise of quantum mechanics. Anger (1964) was the first to use it for simulating the physical response of a new scintillation camera. Since then, a wide variety of problems in nuclear medicine have been tackled using Monte Carlo simulations (Zaidi 1999, Ljungberg et al 1998, Du et al 2002). The goals mainly were the optimization of new camera designs, the evaluation of correction and reconstruction techniques and the development of new methods for improving image quality in order to favour quantitation. Monte Carlo simulations are also often applied in therapeutic applications (Andreo 1999, Zaidi and Sgouros 2002).

Dedicated codes exist to address problems specific to single photon emission computed tomography (SPECT) such as SimSET (Harrison et al 1993), SIMIND (Ljungberg and Strand 1989), SimSPECT (Yanch et al 1992) and to positron emission tomography (PET) such as PETSIM (Thomson et al 1992) and Eidolon (Zaidi et al 1999). One may also use general purpose nuclear physics codes such as EGS4 (Nelson et al 1985), Geant4 (Agostinelli et al 2002) or MCNP (Briesmeister et al 1988). The main advantages of these general purpose codes are: they are widely used and extensively tested, they can be regarded as long-term existent as well as supported, and they are continuously evolving and therefore use the best of current hardware and software capabilities. There are fewer limitations on their possible applications, and fewer simplifying assumptions are made: for example, the processes taking place in the collimator can be thoroughly simulated whereas dedicated codes often use a parametric model. Their non-specific design also offers the opportunity to implement future developments in SPECT and PET cameras (Buvat and Castiglioni 2002), whereas dedicated codes are limited by the time of their design.

Therefore, GATE was designed as an upper layer for the Geant4 nuclear physics code and was tuned for use in nuclear medicine, more specifically to fulfil its role as a simulation platform for PET and SPECT (Santin et al 2002). GATE thus incorporates all Geant4 features including well-validated physics models, geometry modelling tools, visualization and a scripting language for commanding the simulation. We developed several new components necessary for SPECT simulations, such as the Interfile image reader for voxelized clinical data, and we modelled parallel hole collimators in which tracking of all scatter processes can be simulated without using simplified geometrical models. The simulations were validated through comparison with experimental data measured on a dual-headed AXIS camera (Philips), which was extensively modelled to represent the physical reality. The validated properties were: spectral distributions, energy resolution, sensitivity, scatter component and spatial resolution analysis. Following the satisfactory results of this validation we simulated a moving point source for transmission imaging $\left(\right.$ Beacon $^{\mathrm{TM}}$ ) to perform a scatter analysis and we modelled a prototype solid state camera design (Solstice) to check sensitivity.

\section{Materials and methods}

\subsection{Basic geometry modelling}

The camera used for validation is the dual-headed AXIS camera, manufactured by Philips Medical Systems. Each detector head contains a $54 \mathrm{~cm}$ transaxial $\times 38 \mathrm{~cm}$ axial NaI crystal of thickness $0.95 \mathrm{~cm}$. The readout is done by photomultiplier tubes (PMTs), diagonally 


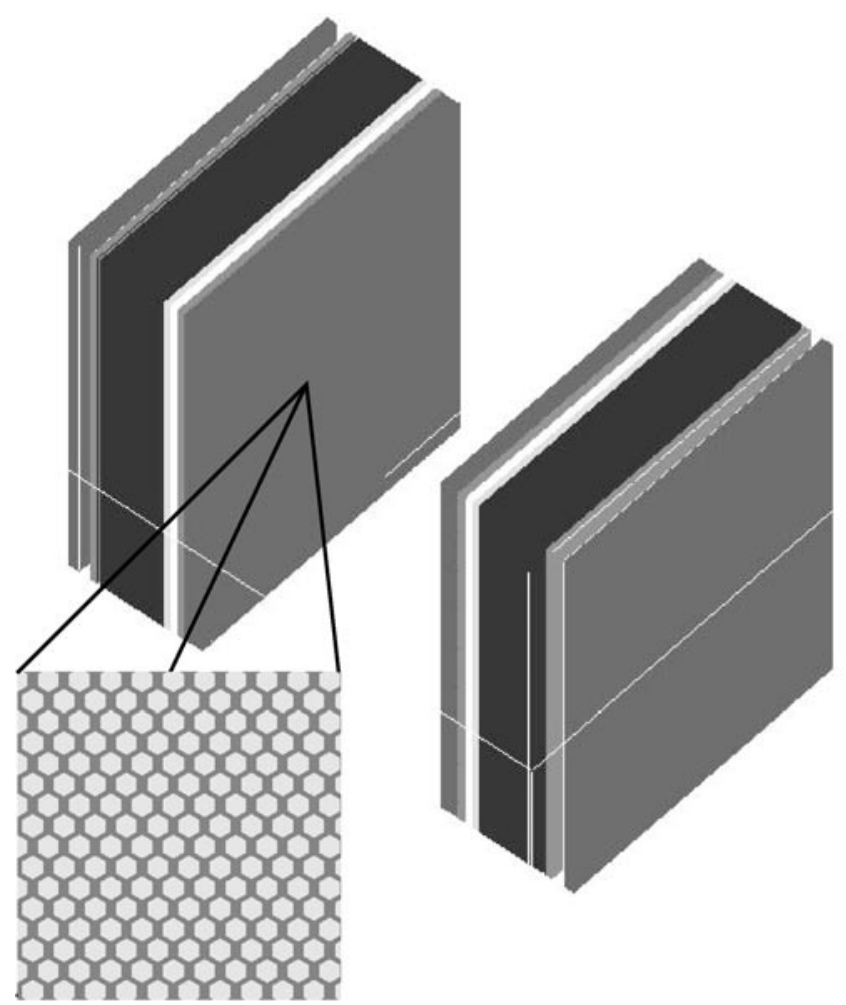

(a)

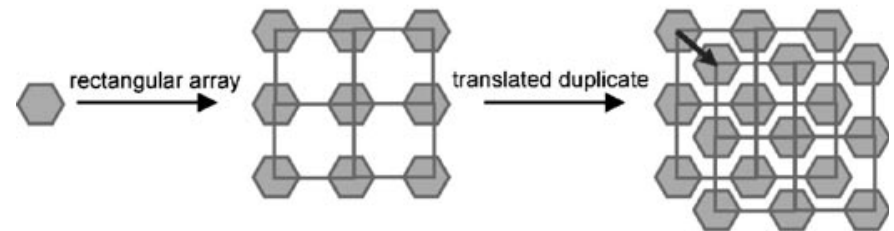

(b)

Figure 1. Detector model: (a) view of the modelled AXIS detector heads with a zoom on the collimator, (b) details of the collimator process.

distributed over the crystal. In optimal conditions the energy resolution at $140.5 \mathrm{keV}$ is $9.5 \%$ and the intrinsic spatial resolution is $0.33 \mathrm{~cm}$.

2.1.1. Collimator. In order to achieve accurate models of low energy high resolution (LEHR) and medium energy general purpose (MEGP) collimators (figure 1(a)), the air holes should be modelled according to the technical specifications concerning the hole diameter and the septal thickness. For this purpose the typical hexagonal shape was added to the GATE geometry package. The construction of the lead collimators was performed by repeating a hexagon on a rectangular array. This array was then filled up with a translated duplicate, creating the complete distribution of air holes, as shown in figure 1(b).

A collimator may be fabricated in two different ways: by casting a mould or by folding plates. In the first case the septa will have the same thickness in all directions while in the 


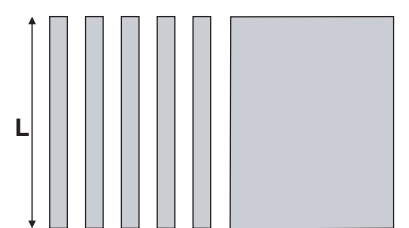

(a)

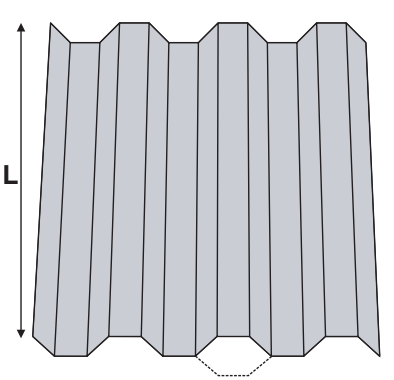

(d)

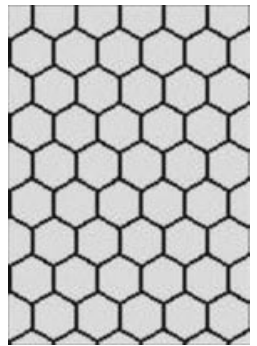

(b)

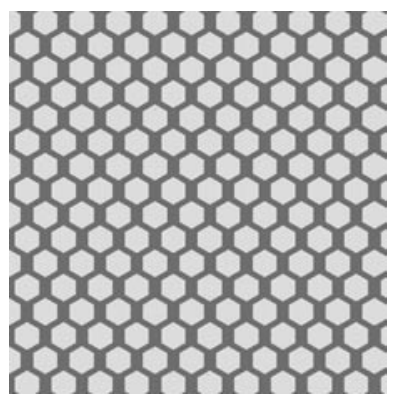

(e)

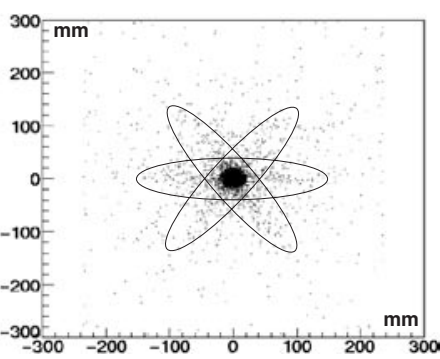

(c)

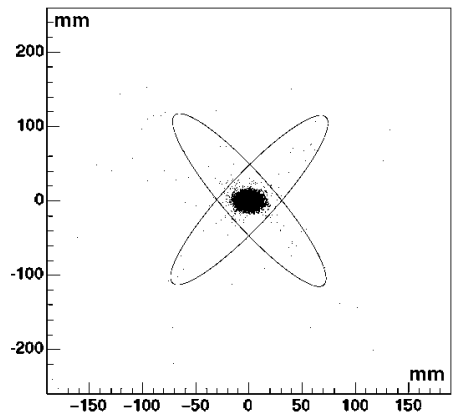

(f)

Figure 2. Simulation of the septal penetration from a point source for two types of collimators: (a) detail cast collimator (length $L$ ); (b) transversal image of a cast collimator; (c) cast collimator penetration; (d) detail collimator plate; (e) transversal image of a folded collimator; (f) folded collimator penetration.

latter case the fabrication process causes the septal thickness along one direction to be twice the thickness along the other directions. The septal penetration will thus be lower along that direction causing the appearance of only four streaks instead of the expected six in point source images. This is demonstrated in figure 2 where a point source was used to simulate the effects of cast collimators and to emphasize the low penetration along one direction for folded collimators. The construction type has implications for the energy spectrum (folding implies weaker x-ray peaks at 70-80 keV), the sensitivity, and the spatial resolution.

When imaging point sources with the LEHR and MEGP collimators, we found four-streak patterns, an effect that was even more obvious when using ${ }^{111} \mathrm{In}$. The folded construction of the collimators was confirmed by acquiring x-ray images of their surfaces. These images were used to complete the specifications of the manufacturer concerning hole radius, septal thickness and collimator length for both collimators.

2.1.2. Detector head design. Figure 3(a) shows a detailed model of the detector head geometry starting from the collimator and incorporating the crystal in its casing before the light guide, the PMTs and the compression plate, further on to the air gap, the lead ending and the shielding. Different materials (aluminium, crown glass, NaI, lead, plastic), linked to their cross sections for photon interaction, were specified in order to provide a realistic model for the AXIS detector head. Additionally, the polymethylmethacrylate (PMMA) plastic material was defined to model Perspex phantoms, needed for evaluation purposes. 


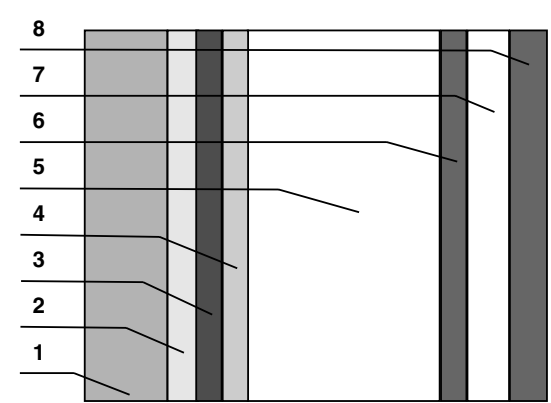

(a)

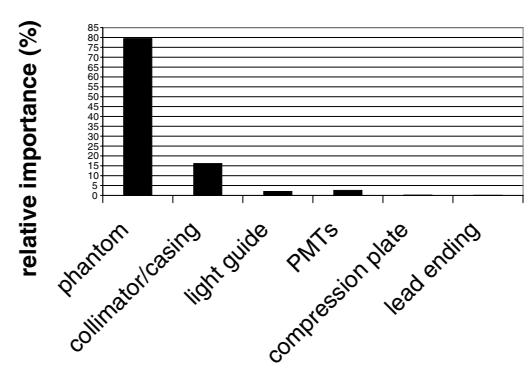

(b)

Figure 3. (a) Axial view of the detector head model with 1: collimator, 2: cover, 3: crystal in its aluminium casing, 4: light guide, 5: PMTs, 6: compression plate, 7: air gap and 8: lead ending (top and bottom shielding of the crystal is omitted for visualization purposes); (b) relative importance of every layer for $140.5 \mathrm{keV}$.

The detector head model was thus composed of an aluminium cover around the NaI crystal of $54 \mathrm{~cm} \times 38 \mathrm{~cm} \times 0.9525 \mathrm{~cm}$. A light guide made of thick crown glass was positioned behind the embedded crystal, followed by the PMTs, modelled as realistically as feasible: glass entrance window, backpart, casings. The back compartment of the detector was ended by an aluminium compression plate, an air gap and a lead shielding covering all three sides of the detector.

Figure 3(b) demonstrates the relative importance of every layer in the model at $140.5 \mathrm{keV}$ by counting the scattered events in each particular part, divided by the total number of scatter events. The phantom used in this simulation is a water-filled cylinder of $20 \mathrm{~cm}$ diameter with a ${ }^{99 \mathrm{~m}} \mathrm{Tc}$ source centred in it. The importance of the backparts of the detector is low but increases with isotope energy. As the AXIS camera is a dual-headed camera both detector heads are present in the simulations to incorporate the backscattering from one head onto the other.

2.1.3. Source geometry. Numerous types of source geometry can be defined as input of simulations by combining typical cylinder, sphere, box, etc structures. Additionally we also implemented an Interfile image reader in order to incorporate voxelized, inhomogeneous, anthropomorphic geometries so that digital phantom or patient data can be used in simulations to reproduce realistic acquisitions. It reads in data stored in Interfile v3.3 format (Interfile 1993). For both cases of source definition and attenuation geometry modelling, dedicated translators are implemented in an object-oriented way. After the emission data are read in for the first case, the grey scales are discretized in numerous intervals, and are then converted to activity levels. Afterwards these activity levels are used to determine the number of primary particles for each voxel. For attenuation geometry modelling based on Interfile data on the other hand, the discretized grey scales of the image are converted to materials, stored in a database. Any material occurring in the study can be built from its elements in advance. After the grey scales are converted to materials, Geant4 libraries are called to allocate the cross sections for each material at any incident energy. An example of a patient attenuation map and its counterpart for simulation purposes is shown in figure 4(a). Figure 4(b) shows an example of the Hoffman brain phantom that is converted into an activity distribution in GATE. We performed a simulation study using the Interfile reader and this digital Hofmann brain phantom. A voxelized source based on the complete brain phantom was included in an initial 

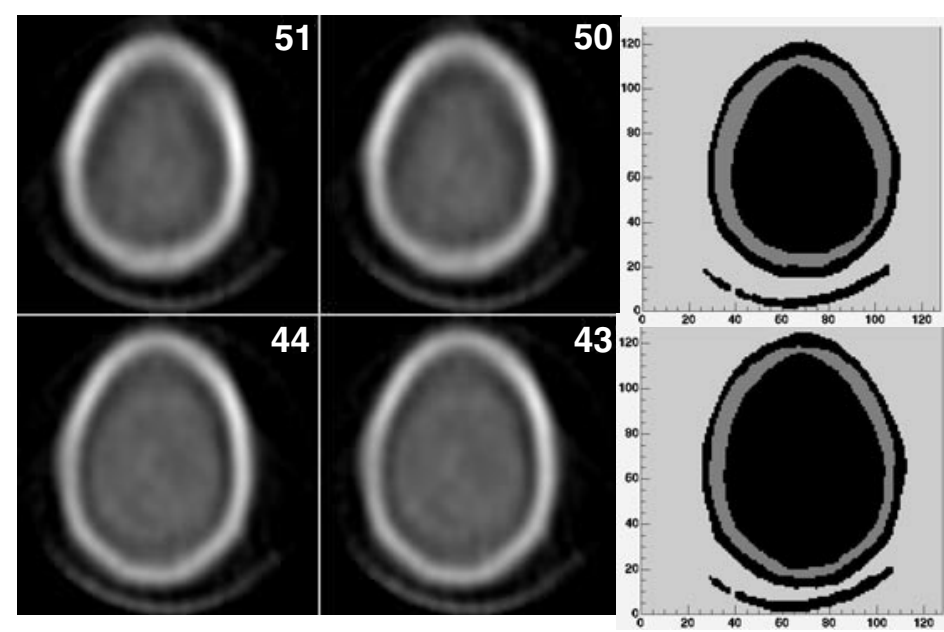

(a)

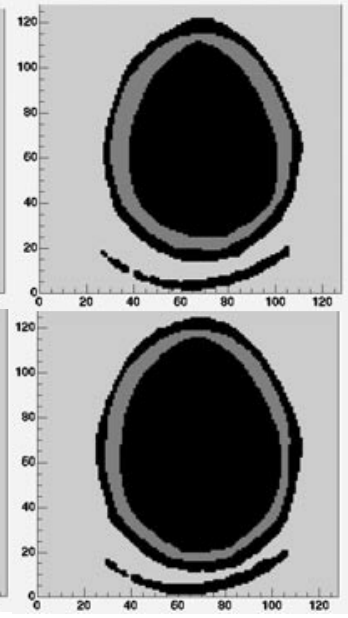

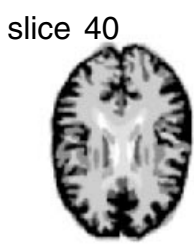

slice 42
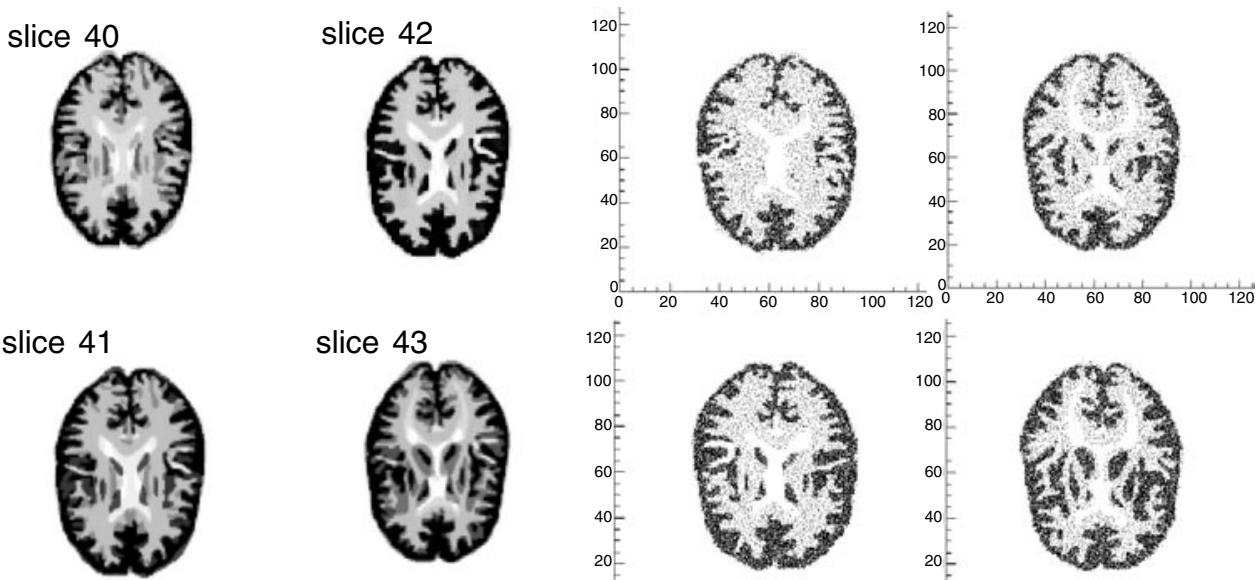

(b)

Figure 4. Clinical extension for an inhomogeneous image: (a) attenuation map from patient data (left: acquisition, right: simulation); (b) emission map from digital Hoffman phantom (left: acquisition, right: simulation).

simulation where we acquired projection data of this source. Afterwards the same digital phantom was used to construct a voxelized attenuation geometry which was incorporated into the simulation. The translation table was built to represent the hardware Hofmann phantom using PMMA and water in several different mixtures according to the grey scale of the image. Simulated sagittal projections of the voxelized source with and without attenuation were then compared.

\subsection{Energy spectra evaluation}

2.2.1. Energy resolution module. The scintillation process and the light detection were not incorporated in our model. An 'energy blurrer' was used instead, introducing a 
Gaussian energy distribution with user-defined mean and standard deviation $\sigma$. We adapted the energy module to obtain an energy-dependent spectral resolution following $\sim 1 / \sqrt{ } E$ (Sorenson and Phelps 1987) with an overall resolution of $9.5 \%$ at $140.5 \mathrm{keV}$, as stated by the manufacturer for the real detector.

The energy resolution at $140.5 \mathrm{keV}$ was confirmed by the following study: real data were taken with a ${ }^{99 \mathrm{~m}} \mathrm{Tc}$ (peak energy at $140.5 \mathrm{keV}$ ) point source of $29 \mathrm{MBq} 15 \mathrm{~cm}$ away from the detector surface (with collimator), and the results were compared to the energy spectrum simulated with GATE in an identical configuration. In a second phase the same source was inserted in a cylindrical water-filled phantom, placed at $10 \mathrm{~cm}$ from the detector. The dimensions of the Perspex cylinder were: $10.75 \mathrm{~cm}$ inner and $11.25 \mathrm{~cm}$ outer radii and $20 \mathrm{~cm}$ height. In both experiments $500 \times 10^{6}$ events were simulated. The spectrum from the pulse height analyser (PHA) was plotted together with the simulation results, normalized to the peak height and analysed with ROOT (Brun and Rademakers 1997). ROOT is an object-oriented data analysis framework with all the functionality needed to handle and analyse large amounts of data in a very efficient way.

2.2.2. Shape of the energy spectra of various isotopes. We also did an evaluation for different isotopes. The energy resolution module described in the previous section was adapted to allow setting the right energy resolution at the appropriate main photopeak energy for each isotope. These energy resolutions were derived from spectra measurements on the camera.

The validation experiments were performed without a collimator, the source of each isotope being placed $20 \mathrm{~cm}$ away from the detector head. Simulations were performed with the same configuration. The spectra visualized with the PHA tool from the acquisition software of the camera were compared to the results of $2 \times 10^{6}$ events, simulated each time. The relative normalization was done by setting the statistical mean of their peaks equal. Evaluation was done for ${ }^{22} \mathrm{Na},{ }^{57} \mathrm{Co}$ and ${ }^{67} \mathrm{Ga}$. A wide range of energies was thus covered as ${ }^{67} \mathrm{Ga}$ has its photopeak around $93 \mathrm{keV},{ }^{57} \mathrm{Co}$ around $122 \mathrm{keV}$ and ${ }^{22} \mathrm{Na}$ around $511 \mathrm{keV}$.

\subsection{Sensitivity validation}

We evaluated the absolute sensitivity (in cps/MBq) of the GATE model of our detector using a ${ }^{57} \mathrm{Co}$ source of $9.3 \mathrm{MBq} \pm 3 \%$. This particular ${ }^{57} \mathrm{Co}$ source is used to calibrate our well counters and therefore its activity was exactly known. We performed a planar acquisition of this source over $600 \mathrm{~s}$ and registered the total number of counts. Dead time correction was not necessary because of the resulting low count rate at the detector. We simulated the same experiment with GATE.

Additionally we evaluated the sensitivity in two separate windows to make sure that there were no additive effects present in the simulations. In the first experiment we performed a static scan (with the LEHR collimator) of a point source ( $1 \mathrm{ml}$ sphere, $2.6 \mathrm{MBq}{ }^{99 \mathrm{~m}} \mathrm{Tc}$ ) at different distances from the detector: $5,15,25,30 \mathrm{~cm}$. We acquired data in the photopeak window (129-151 keV) and in the Compton window (92-126 keV). We set up corresponding simulations for each experiment and we compared the simulated and experimental sensitivities. Error bars were added due to the imprecision on the activity of the ${ }^{99 \mathrm{~m}} \mathrm{Tc}$ source. For each simulation $150 \times 10^{6}$ events were tracked. We repeated a similar experiment with the MEGP collimator where the same source was used and acquisitions were made at $0,15,30$ and $45 \mathrm{~cm}$ from the detector. Again the experiments were reproduced using GATE. Errors bars, deduced from Poisson statistics, were added to the simulation results. 


\subsection{Scatter profiles}

We extended the validation by acquiring scatter profiles at different distances from the detector, with and without attenuation, in the Compton (92-126 keV) and photopeak window (129-151 keV). In the first experiment we performed a static scan of a point source $(1 \mathrm{ml}$ sphere, $2.6 \mathrm{MBq}^{99 \mathrm{~m}} \mathrm{Tc}$ ) in air at 5, 15, 25, $30 \mathrm{~cm}$ from the detector with the LEHR collimator. This was done for the photopeak window and for the Compton window. In a second experiment the point source was replaced by a line source $\left(0.1 \mathrm{~cm}\right.$ diameter, $10 \mathrm{~cm}$ long, $\left.2 \mathrm{MBq}{ }^{99 \mathrm{~m}} \mathrm{Tc}\right)$ placed in a water-filled cylindrical phantom of $22.3 \mathrm{~cm}$ diameter at $10 \mathrm{~cm}$ from the detector heads with the LEHR collimators attached. Acquisitions were made in the photopeak window at both detector heads for the line source in the centre of the phantom, at $1 \mathrm{~cm}, 5 \mathrm{~cm}$, $10 \mathrm{~cm}, 15 \mathrm{~cm}$ and $20 \mathrm{~cm}$ depth. For each simulation $500 \times 10^{6}$ events were tracked and the simulated scatter profiles were compared with experimental acquisitions through a best fit plot.

\subsection{Spatial resolution comparison}

A 'spatial blurrer' module was created to model the intrinsic spatial resolution, caused by crystal scatter and signal readout, while the extrinsic resolution (caused by all other parts of the detector) was simulated. The spatial blurrer replaces the recorded position of every count in the crystal by a Gaussian blurred position, in the same way as shown in section 2.2.1 for the energy resolution. This is a reasonable assumption, based on the fact that at $140.5 \mathrm{keV}$ ( ${ }^{99 \mathrm{~m}} \mathrm{Tc}$ was used in the following experiments) the scatter level in the crystal is very low: only $17 \%$ of the interactions in the crystal are due to scatter, which influences mostly the full width at tenth maximum (FWTM) rather than the spatial resolution, i.e. the full width at half maximum (FWHM). The FWTM is only used as a parameter to provide a second measure because the curve deviates slightly from a true Gaussian. The actual value of the intrinsic resolution was set in the simulations according to the estimate given by the manufacturer $(0.33 \mathrm{~cm})$. The contribution of the collimator to the spatial resolution was fully simulated.

In order to check the validity of both the spatial blurrer for the intrinsic resolution and the collimator model, experiments were conducted. We performed scans of a line source phantom of $0.1 \mathrm{~cm}$ diameter and $5 \mathrm{~cm}$ height, filled with $19 \mathrm{MBq}{ }^{99 \mathrm{~m}} \mathrm{Tc}$, at different distances from the detector with the LEHR collimators attached: 5, 10, 20 and $40 \mathrm{~cm}$. The FWHM of each acquisition was determined. The same procedure was used for the simulations where $20 \times 10^{6}$ events were simulated each time, followed by a ROOT analysis for determination of the FWHM (figure 5). This was repeated for a line source of $0.2 \mathrm{~cm}$ diameter filled with $66 \mathrm{MBq}{ }^{99 m} \mathrm{Tc}$ at 5, 15, 27, 35 and $45 \mathrm{~cm}$ from the detector with the MEGP collimators attached to it. Finally, we extended the LEHR experimental setup by inserting the line source in the centre of a water-filled phantom with the same specifications as in section 2.2.1. This phantom was placed at $10 \mathrm{~cm}$ from the detector head so that the line source was at $21 \mathrm{~cm}$ from the detector. FWHM measurements were repeated for error bar determination while the error bars on the simulation results were derived from the ROOT fitting procedure.

\subsection{Applications}

We applied GATE to two realistic situations to demonstrate the advantages of a general purpose simulation package, where no geometrical approximations are made and where all physical processes are taken into account. 


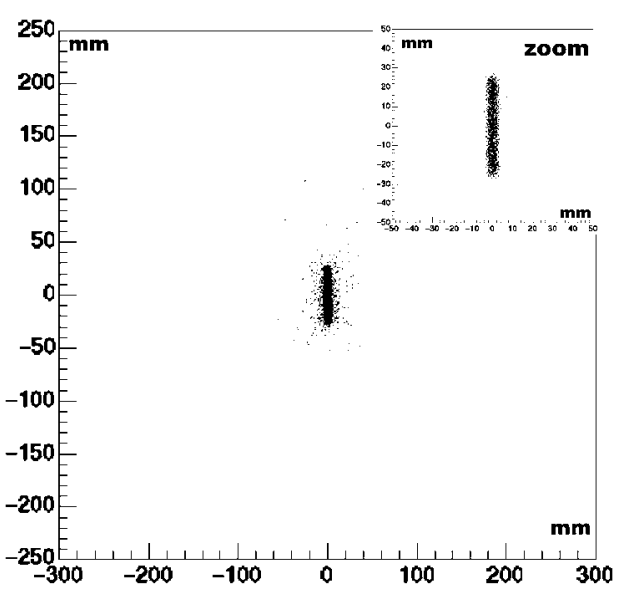

(a)

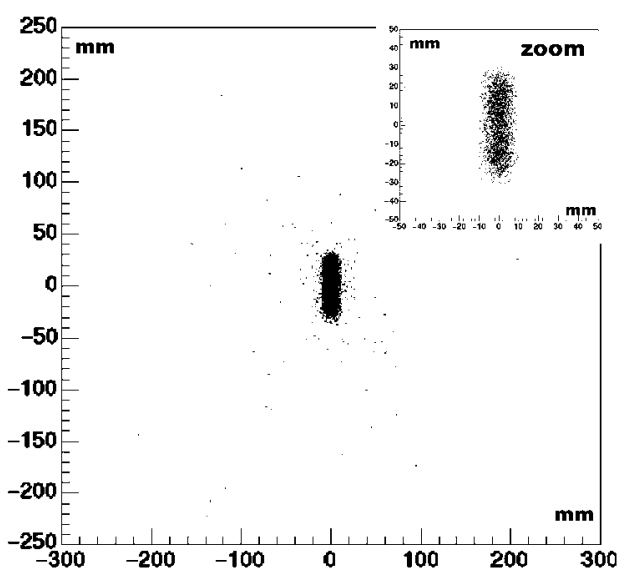

(c)

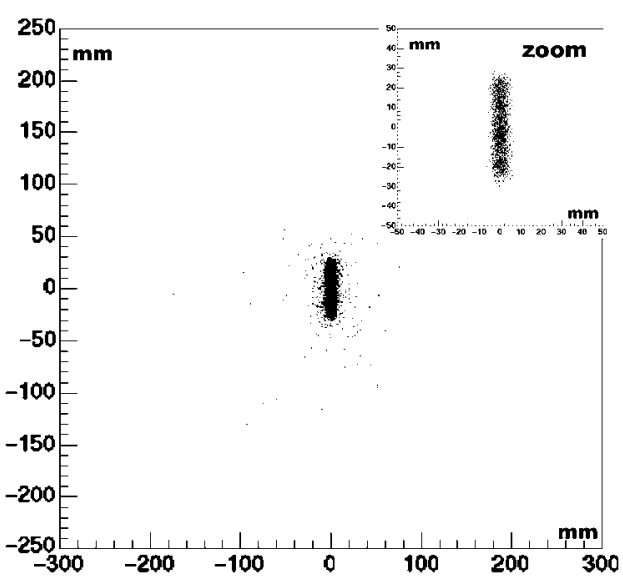

(b)

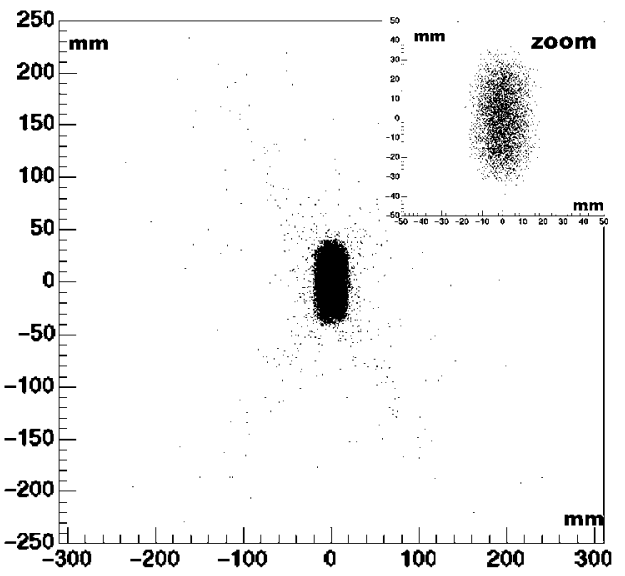

(d)

Figure 5. Simulated acquisitions of $0.1 \mathrm{~cm}$ line source in air at different distances from the detector surface for the LEHR collimator: (a) $5 \mathrm{~cm}$; (b) $10 \mathrm{~cm}$; (c) $20 \mathrm{~cm}$; (d) $40 \mathrm{~cm}$. The plane shown is the physical FOV and a detail of the centre is provided.

2.6.1. Beacon attenuation correction. The first test consisted in modelling the Beacon ${ }^{\mathrm{TM}}$ attenuation correction tool attached to the AXIS detector heads (Zeng et al 2001b). It is made of moving ${ }^{133} \mathrm{Ba}(356 \mathrm{keV})$ point sources of $370 \mathrm{MBq}$ that irradiate the opposite detector head through the collimator to form transmission images (figure 6). The usage of GATE in protocol optimization is demonstrated by investigating the scatter order of the photons from the Beacon source reaching the detector, and by investigating the scatter contributions from the different detector components for different energy windows. Additionally the scatter of the Beacon source photons in the transmission window (302-409 keV) and the degrading downscatter on the ${ }^{99 m} \mathrm{Tc}$ emission photopeak window (129-151 keV) in simultaneous emission and transmission scanning are investigated.

2.6.2. Solstice detector. A case study was performed to demonstrate the use of GATE in camera design. It consisted in modelling the prototype of the solid state with compact 


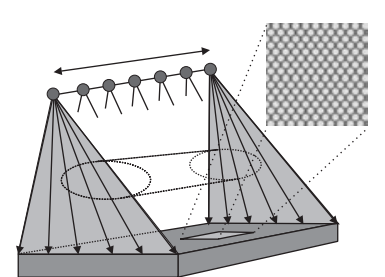

(a)

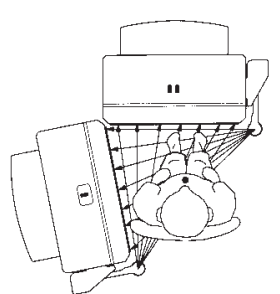

(b)

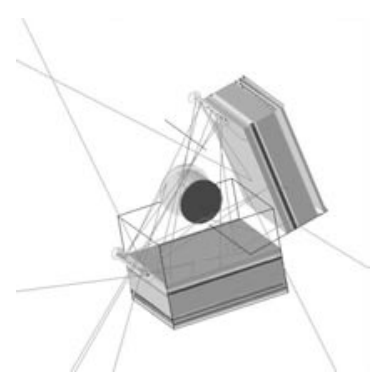

(c)

Figure 6. Beacon: (a) moving ${ }^{133} \mathrm{Ba}$ source irradiating the opposite detector; (b) illustration of the Beacon device; (c) model of this setup: two detectors with Beacon devices attached, rotating around a cylindrical phantom with 100 photon tracks included as an illustration.

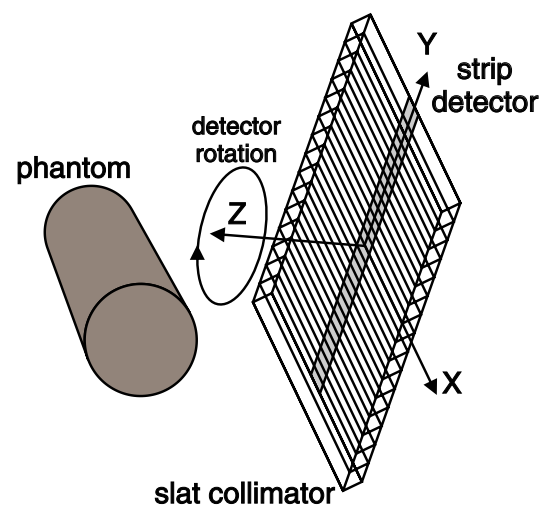

(a)

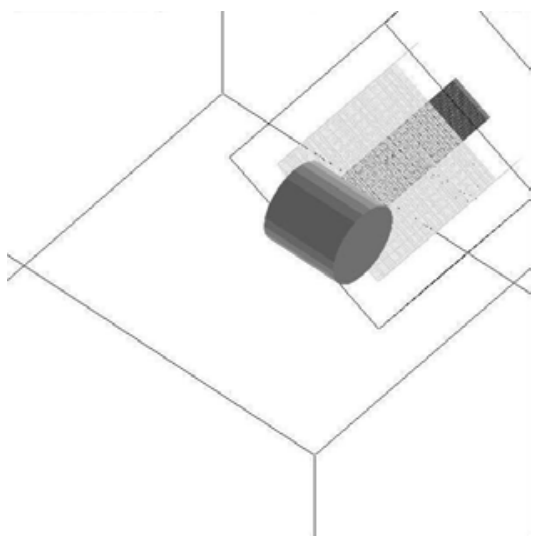

(b)

Figure 7. Solstice: (a) illustration of the Solstice detector; (b) model of this setup: Solstice detector rotating in front of the cylindrical phantom: CZT strip and collimator slats visualized.

electronics (Solstice) camera (figure 7(a)), (Gagnon et al 2001, Griesmer et al 2001). The concept of the Solstice detector consists of a rotating slat collimator mounted on a strip detector $(35 \mathrm{~cm} \times 5.6 \mathrm{~cm} \times 0.5 \mathrm{~cm})$, made of cadmium zinc telluride $(\mathrm{CZT})$, and subdivided into an array of 88 crystals. The collimator slats were made of tungsten and lead and have a pitch of $0.18 \mathrm{~cm}$ and a thickness of $0.03 \mathrm{~cm}$. The detector assembly is spinning to get 2D images and revolves around the object for $3 \mathrm{D}$ reconstruction. The main purpose of this new design is to improve the resolution versus sensitivity tradeoff by the rotating collimator, coupled to an improved energy resolution by using the semiconductor detector. Typical experimental values are $5 \%$ energy resolution at $140 \mathrm{keV}$ and a spatial resolution of $0.5 \mathrm{~cm}$ at $10 \mathrm{~cm}$ from the detector. We modelled a rotating slat collimator in GATE (figure 7(b)) on a CZT strip with the correct pixelization. Every physical interaction, except for the electron hole trapping, was modelled. Afterwards we compared our simulated sensitivity against theoretical calculations for 30 point sources of ${ }^{99 \mathrm{~m}} \mathrm{Tc}$ placed on an arc with an aperture of $90^{\circ}$ so that the distance to the detector stayed constant for each sphere of the phantom. 


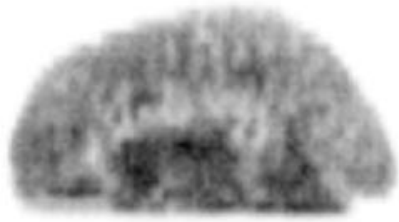

(a)

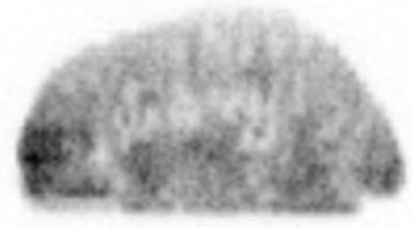

(b)

Figure 8. Interfile reader: simulated projection data of the Hofmann brain phantom: (a) without attenuation; (b) with a voxelized attenuation geometry based on the same phantom.

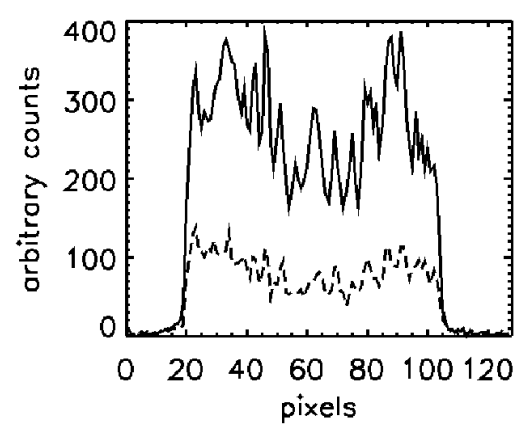

Figure 9. Midway horizontal profile of the simulated voxelized source sagittal projection data with (dashed line) and without (full line) the voxelized attenuation geometry.

\section{Results}

\subsection{Basic modelling}

Some initial simulations were performed to demonstrate the usefulness of the voxelized source and the voxelized attenuation geometry modelling. We simulated projection data of the Hofmann brain phantom with and without a voxelized attenuation geometry (figure 8). The attenuation geometry material definition is the analogon of the hardware Hofmann phantom using the dedicated implemented grey level to material definition translator. To demonstrate the effect of the attenuation, both projection data are compared by plotting two midway horizontal profiles of the sagittal projections as shown in figure 9. The voxelized nature of the results is obvious, as well as the appropriate attenuation effect.

\subsection{Energy spectra}

In figure 10(a) the simulated spectrum and the acquired spectrum of a ${ }^{99 \mathrm{~m}} \mathrm{Tc}$ source in air at $15 \mathrm{~cm}$ from the detector are compared. Good agreement was found regarding the position and height of the photopeak and of the lead peak. Slight differences may be observed at some points but these discrepancies remain fairly limited. Figure 10(b) visualizes the spectrum of a radioactive ${ }^{99 \mathrm{~m}} \mathrm{Tc}$ source in an attenuating and scattering medium at $10 \mathrm{~cm}$ from the detector. The simulation and the experiment fully agree on the increased contribution at lower energies, caused by photons that were scattered in the water-filled phantom. The spectral distributions for various other isotopes are summarized in figure 11 as the result of another experiment where no collimators were attached to the detectors. For all these isotopes, showing various energy 


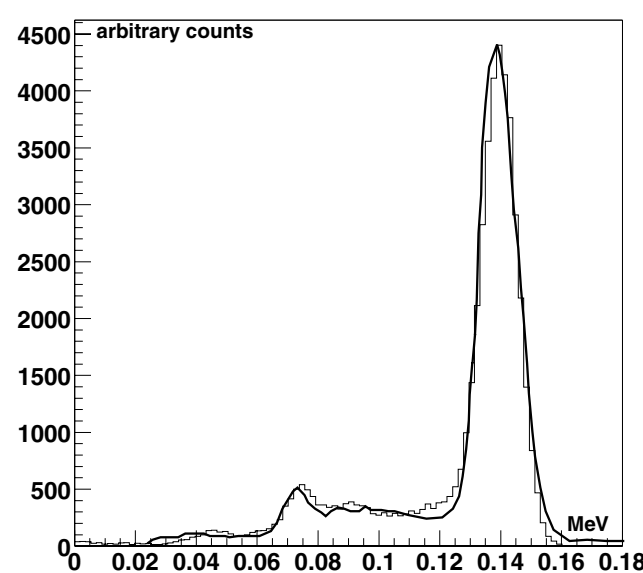

(a)

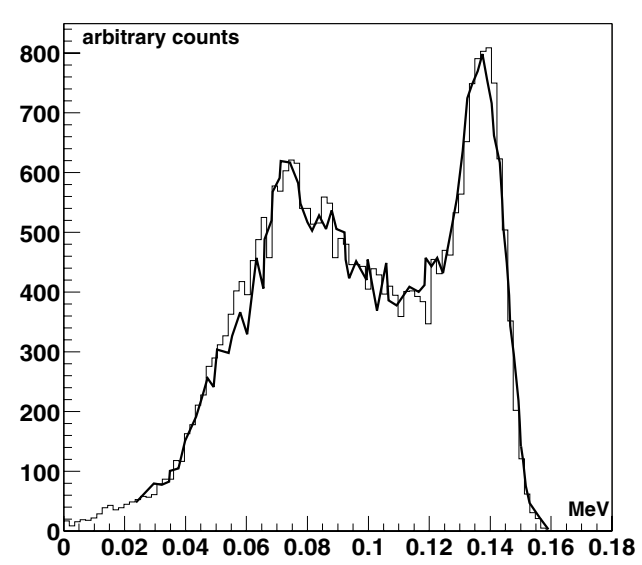

(b)

Figure 10. Comparison of simulated and measured ${ }^{99 \mathrm{~m}} \mathrm{Tc}$ spectrum: (a) source in air; (b) with $\mathrm{H}_{2} \mathrm{O}$ scattering medium. Black line: measurement, grey line: binned simulation.

distributions and photopeak energies, the experimental spectral distributions are perfectly reproduced by the simulations.

\subsection{Sensitivity}

The absolute sensitivity measured with the ${ }^{57} \mathrm{Co}$ source was $231 \mathrm{cps} / \mathrm{MBq}$, this value being quite reliable since we used a high-precision calibration source. The simulated absolute sensitivity was $246 \mathrm{cps} / \mathrm{MBq}$, showing a difference of only $6.1 \%$ with the experimental value. In this experiment no energy windows were set and the possibility existed that processes were overestimated in one energy range and underestimated in another. Inter-window error compensation was ruled out however by performing experiments that measure the sensitivity in two separate smaller windows. In figure 12(a) the results are shown for a study with a ${ }^{99 \mathrm{~m}} \mathrm{Tc}$ source in air on the LEHR collimator for the photopeak and the Compton window. We see good agreement between the experiments and the simulations within the range of the error bars that arise from the uncertainty in the activity determination of the ${ }^{99 \mathrm{~m}} \mathrm{Tc}$ source for the experiment and from Poisson statistics for the simulations. The results of the analogous MEGP experiments are shown in figure 12(b) where again the sensitivity in both energy windows is plotted, demonstrating good agreement between simulations and reality.

\subsection{Scatter profiles}

Simulated point spread functions of a point source in air for the photopeak window are plotted in figure 13 as an indication of the effect of distance on the FWHM. Figure 14 shows the simulated and measured scatter profiles of point sources in air for the Compton window (92-126 keV) at different distances, thus evaluating the simulation of scatter within the detector head. The good sensitivity agreement in the Compton window for the LEHR and MEGP collimators (section 3.3) was a first indication of the correct simulation of scattered events in the collimator and in the other parts of the detector head as there was also no phantom present in that particular sensitivity experiment. The scatter profiles for the Compton window 


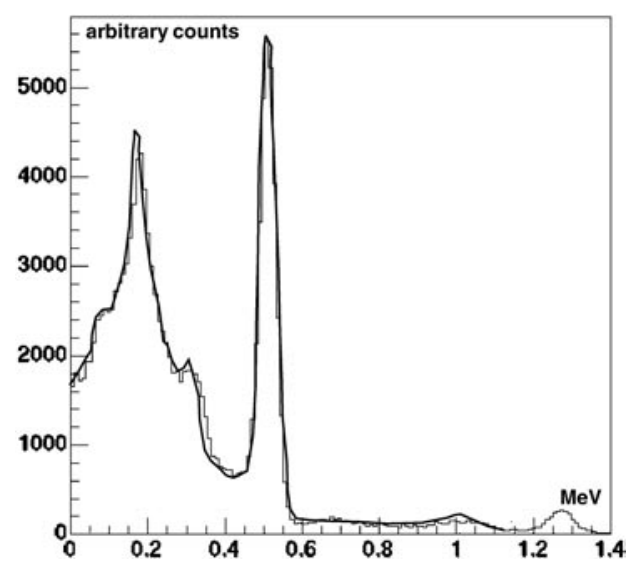

(a)

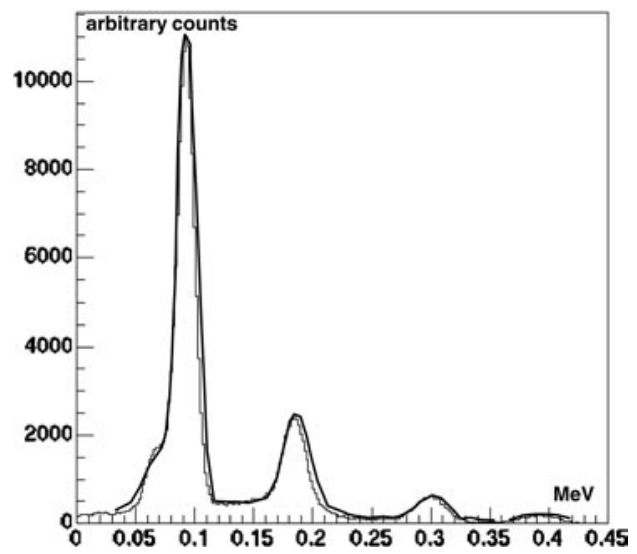

(c)

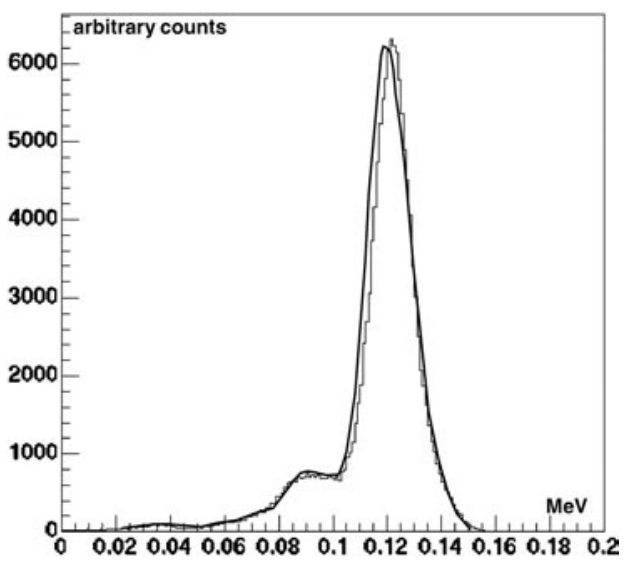

(b)

Figure 11. Comparison of simulated and measured spectra in air: (a) spectrum of ${ }^{22} \mathrm{Na}$; (b) spectrum of ${ }^{57} \mathrm{Co}$; (c) spectrum of ${ }^{67} \mathrm{Ga}$. Black line: measurement, grey line: binned simulation.

confirm this expectation as can be concluded from the good agreement between experiment and simulation.

To evaluate the scatter in a phantom we performed a second experiment and scatter profiles of a line source at different depths in an attenuating environment for the photopeak window were acquired. This is shown in figure 15. We may thus conclude that the scatter processes in the phantom are appropriately modelled for all cases and that the FWHM increases with depth, as expected.

\subsection{Spatial resolution}

Figure 16 shows the result of the spatial resolution experiments where the simulated FWHMs are compared with the measured FWHMs of line sources in air. A good agreement is reached 


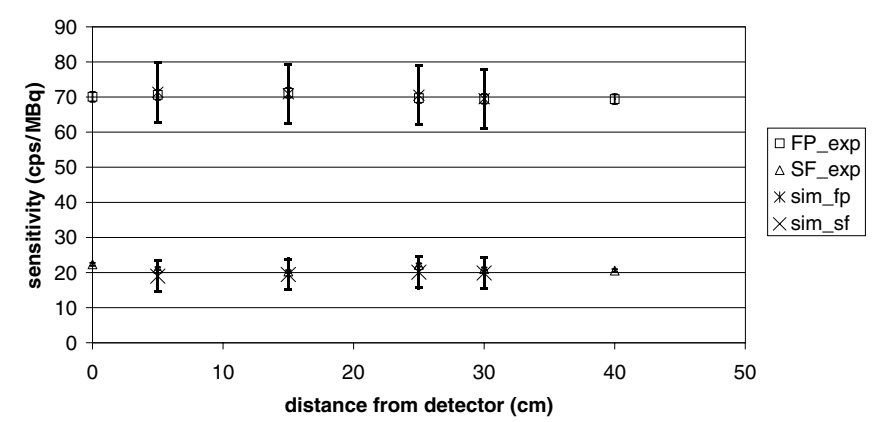

(a)

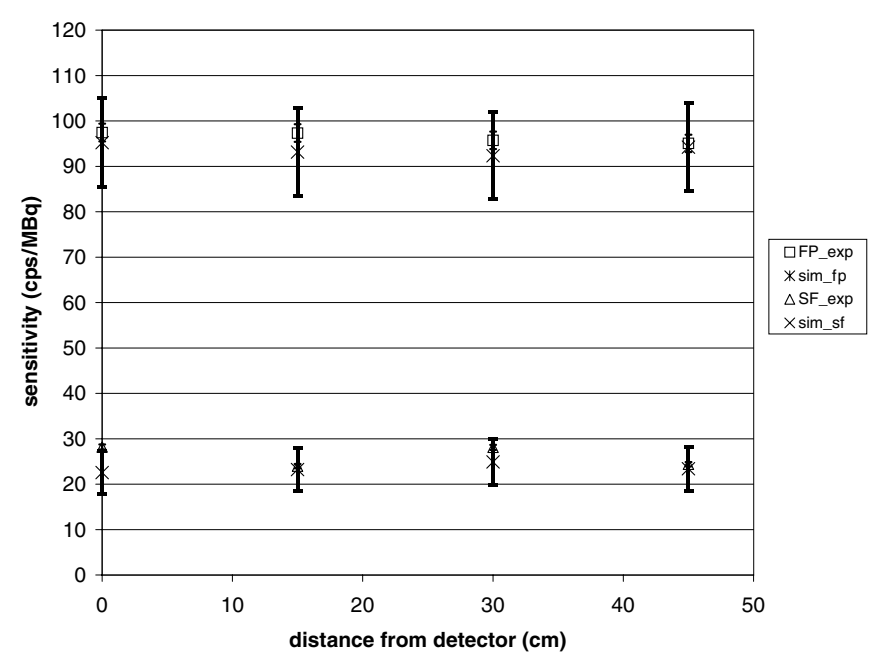

(b)

Figure 12. Sensitivity: (a) LEHR collimator; (b) MEGP collimator. FP = photopeak window (129-151 keV), SF = scatter in the 92-126 keV range (Compton window).

within the error bars, both for the LEHR and for the MEGP collimators. When the source was placed in the centre of an attenuating geometry, we found an FWHM of $1.3 \mathrm{~cm} \pm 0.1 \mathrm{~cm}$ on the real data, while this was $1.36 \mathrm{~cm} \pm 0.02 \mathrm{~cm}$ in the simulation.

\subsection{Applications}

3.6.1. Beacon. Figure 17(a) represents the scatter order of the detected photons in a typical Beacon simulation for different energy windows. A scatter order equal to one means that the detected photon had scattered once before reaching its detection location. Of all detected photons, $35 \%$ reached the crystal without scattering while $27 \%$ scattered once. For the main peak window $71 \%$ of all detections were primaries while $23 \%$ were first-order scatter events. Multiple scattered photons contribute to the resulting spectrum to a large extent. Figure 17(b) details which elements of the setup caused this scatter by counting the scatter events in each part, divided by the total number of scatter events (relative importance). The largest contribution comes from the collimator while the PMTs and the light guide are the second most important parts if one takes the whole spectrum into account. When the energy range is limited to the transmission energy window the collimator is the most important contributor 


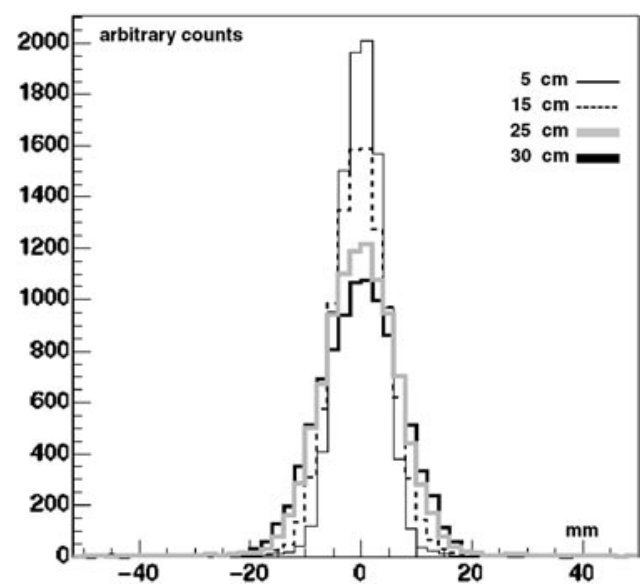

Figure 13. Simulated scatter profiles of a point source in air for the photopeak window at different distances from the LEHR collimator.

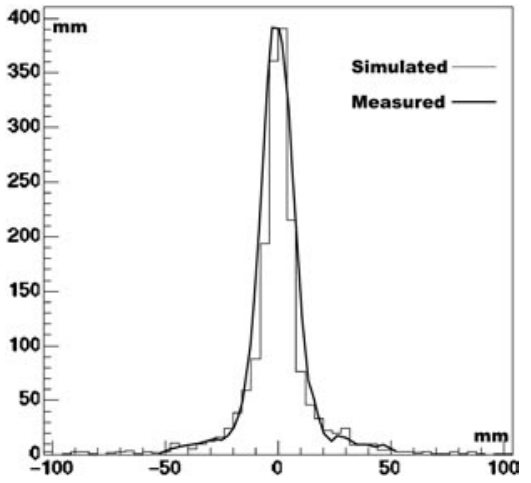

(a)

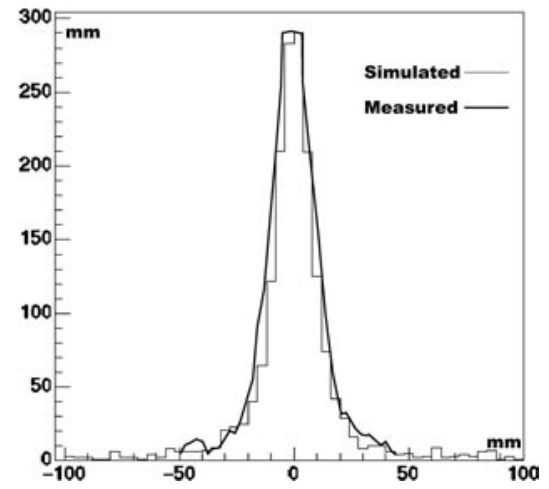

(c)

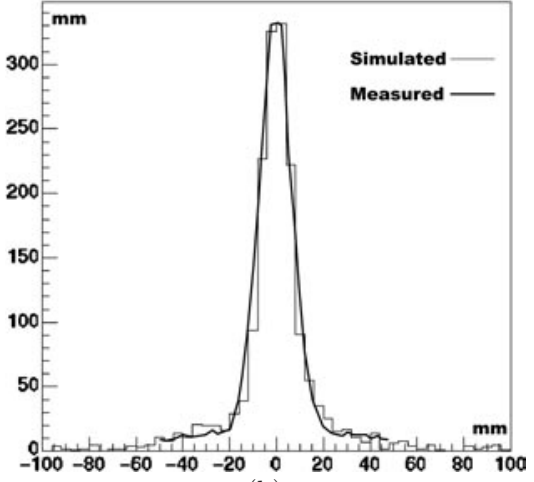

(b)

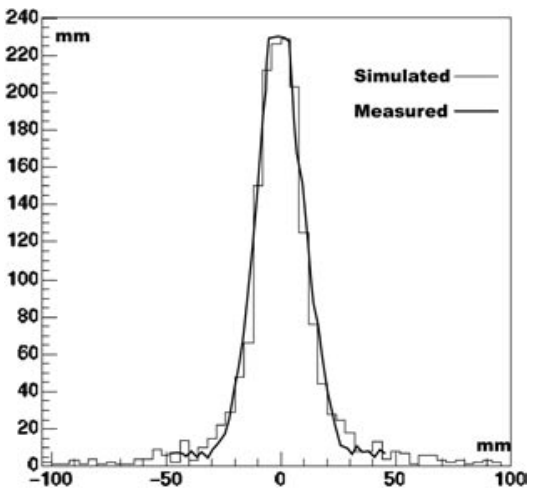

(d)

Figure 14. Simulated and measured scatter profiles of a point source in the Compton window in air: (a) $5 \mathrm{~cm}$; (b) $15 \mathrm{~cm}$; (c) $25 \mathrm{~cm}$; (d) $30 \mathrm{~cm}$. LEHR collimator attached. 


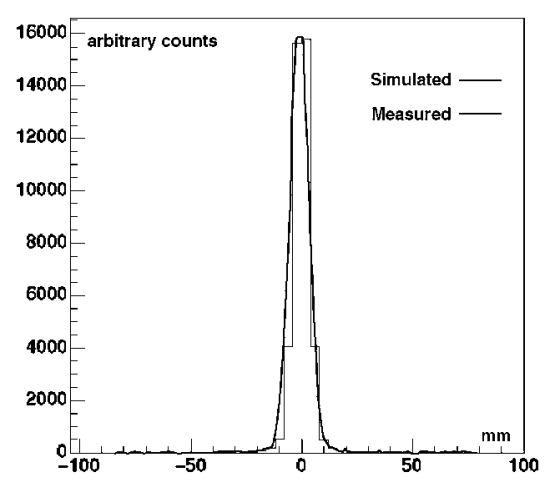

(a)

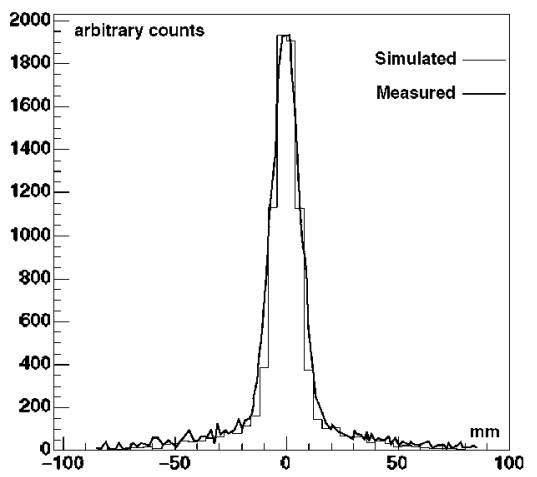

(c)

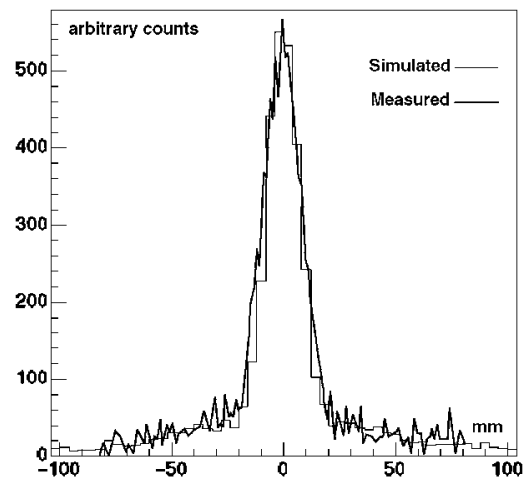

(e)

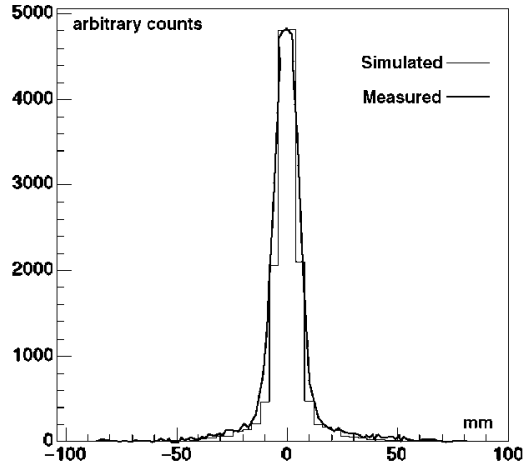

(b)

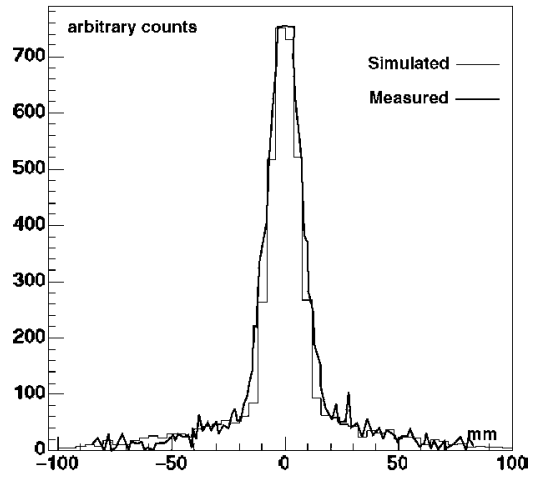

(d)

Figure 15. Simulated and measured scatter profiles of a line source in the photopeak window with $\mathrm{H}_{2} \mathrm{O}$ scattering medium: (a) $1 \mathrm{~cm}$ depth; (b) $5 \mathrm{~cm}$ depth; (c) $10 \mathrm{~cm}$ depth; (d) $15 \mathrm{~cm}$ depth; (e) $20 \mathrm{~cm}$ depth. LEHR collimator attached.

for scattered events while this is the light guide and the PMTS for the emission energy window. From figure 17(c) we can conclude that the scattered photons from the Beacon source contaminate the attenuation energy window (303-409 keV) and that the emission energy window around $140.5 \mathrm{keV}(129-151 \mathrm{keV})$ will be affected by the downscattered ${ }^{133} \mathrm{Ba}$ photons if we apply the device in simultaneous emission and transmission mode. 


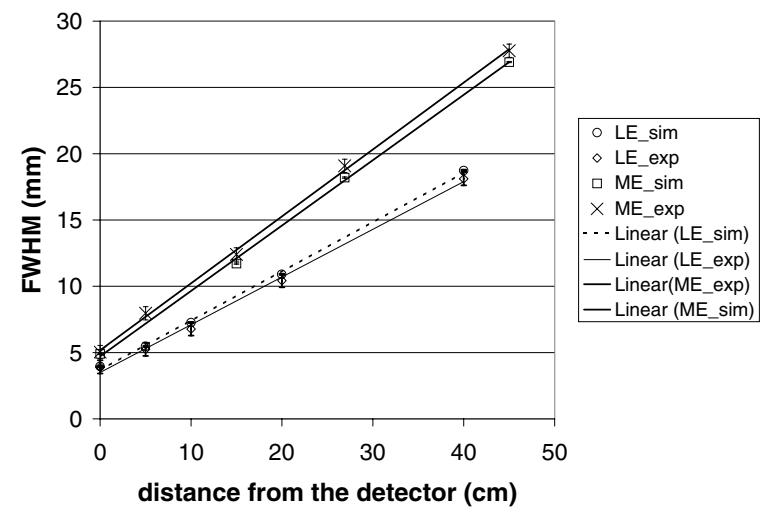

Figure 16. Comparison of spatial resolution for a LEHR and for a MEGP collimator (LE = low energy, $\mathrm{ME}=$ medium energy, $\operatorname{sim}=$ simulated values, exp $=$ experimental values): LE_sim shows the simulated spatial resolution, LE_exp shows the acquired spatial resolution for the LEHR collimator while ME_sim and ME_exp illustrate simulated and acquired spatial resolution for the MEGP collimator; a linear curve is fitted to each data plot.

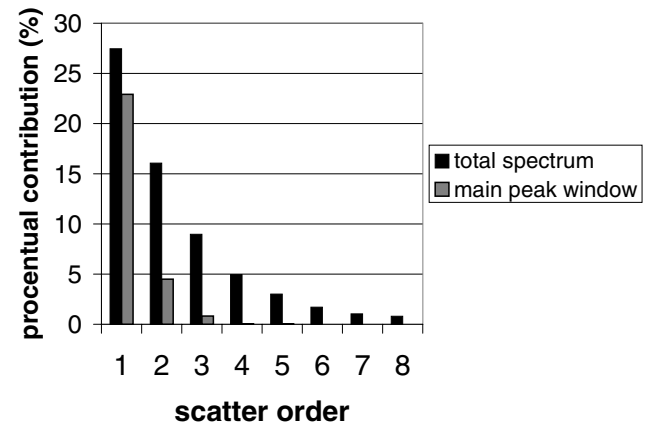

(a)

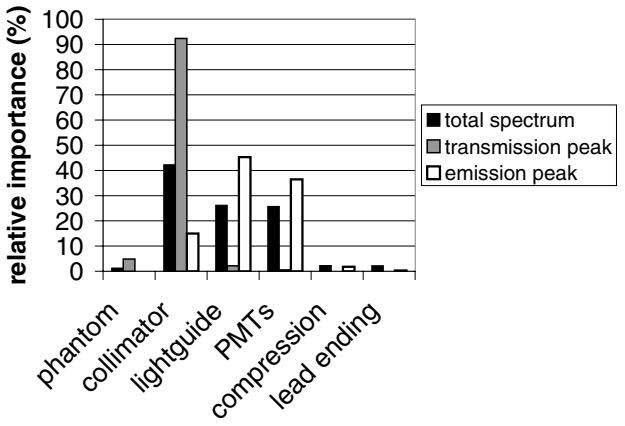

(b)

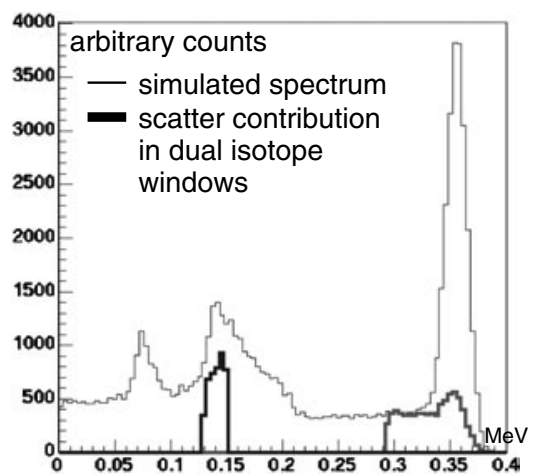

(c)

Figure 17. Simulation results of Beacon: (a) scatter order; (b) relative importance of every layer at $356 \mathrm{keV}$; (c) spectrum: scatter contamination.

3.6.2. Solstice. Our model of the Solstice prototype was evaluated by checking its sensitivity at a predefined distance $r$ from the collimator. As described in the literature (Zeng et al 2001a), 


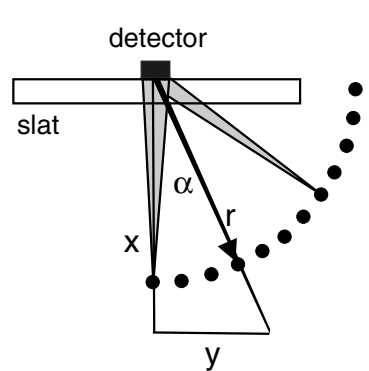

(a)

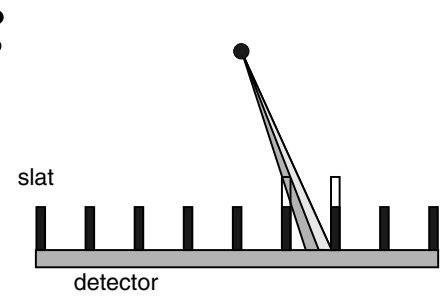

(b)

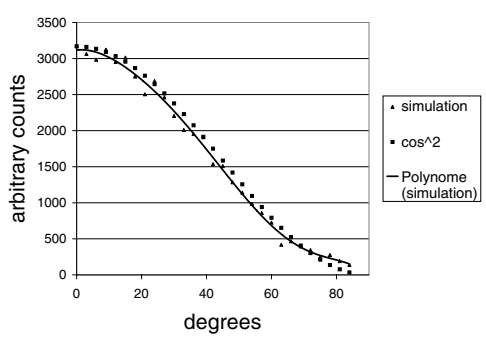

(c)

Figure 18. Evaluation of the simulated Solstice sensitivity at a constant distance from the detector: (a) oblique irradiation effect; (b) slats longer for oblique incidences; (c) comparison simulated versus experimental values.

the sensitivity at a certain distance $r$ should vary as $\cos ^{2}(\alpha)$, with $(\alpha, r)$ being the polar coordinates in the plane perpendicular to the detector. The setup, incorporating 30 spheres with a $3^{\circ}$ angular separation placed on an arc, provided the number of detected counts for each source separately, in order to form the sensitivity variation. The highest sensitivity was registered for point sources in front of the detector while the sensitivity decreases with $\cos ^{2}(\alpha)$ for oblique incidence. The non-perpendicular radiation on the detector (figure 18(a)) causes the sensitivity to decrease for sources at $\alpha \neq 0$. Also for these sources the total collimator slat length is longer due to the oblique path which causes an additional decrease (figure 18(b)). Figure 18(c) compares the theoretical predictions from literature with the simulated result (polynomial fit) and good agreement can be observed.

\subsection{Calculation time}

Simulations have been done on an $850 \mathrm{MHz}$ PC, a $1 \mathrm{GHz}$ PC, one dual $2.6 \mathrm{GHz}$ PC and on a cluster of 11 nodes of which five were Celeron $500 \mathrm{MHz}$ processors and six were AMD Athlon $1.1 \mathrm{GHz}$ processors. An average of 500 events/s was reached when incorporating all processes in the collimator. When replacing the collimator by a simplifying geometrical model the simulation speed was increased to 33.6 s for a 10000 counts energy spectrum $(3 \times$ $10^{6}$ events/s) compared to 10-20 s for a code such as SIMIND.

The reasons for the high computation time in the simulations with collimator are fourfold. The total number of registered counts in SPECT is less than $0.02 \%$ of the generated events because the collimator in front of the crystal stops most of the incoming photons and registers all processes present. This effect is most pronounced with the LEHR variant of the collimator. Secondly, GATE tracks every photon through every object of the experiment, and the LEHR collimator for instance consists of 161120 individual air holes. Ongoing research is being performed on parametrized replicas for the collimator hole arrays that will speed up the simulations by a factor of 10 to 100 . Thirdly, no variance reduction methods were used so far although future research is planned on this topic. Finally, the emission angle was not limited to a certain range so as not to influence the scatter processes in the phantoms or the backscattering from the second detector head. In some experiments, however, computation time could be reduced by replacing the disintegration scheme by a source of monoenergetic gammas as the time needed for the sampling of the disintegration scheme is omitted in this way. The precision of the simulations decreases however with this approach as only the main decay is modelled. 


\section{Discussion}

One of the most important parts of a scintillation camera is the collimator. Its fabrication and constitution are important for the results achieved with the geometrical description of that detector in Monte Carlo simulations. To complete the technical specifications from the manufacturer and to have accurate dimensions, $\mathrm{x}$-ray images of the collimators were taken. We concluded that the collimators were folded rather than cast, causing the septal thickness to be double along one direction. With this folded collimator geometry incorporated in the simulator, the appropriate collimator penetration and lead-interactions were registered on the crystal, as could be concluded from the energy spectra around 70-80 keV (x-ray peak of lead). If we replaced the folded collimator model by the inadequate cast model, then an overshoot around this peak would occur due to the overestimation of the septal penetration along one direction causing six-streak patterns instead of the acquired four-streak patterns.

The importance of the other parts of the detector in the simulations is represented in figure 3(b). The end parts become more important as the photopeak energy of the isotope increases as shown by the comparison with figure 17(b). In this particular case the photons emitted by the ${ }^{133} \mathrm{Ba}$ source have an energy of $356 \mathrm{keV}$ that enables them to penetrate the collimator material while this is not possible for the photons from the ${ }^{99 \mathrm{~m}} \mathrm{Tc}$ source.

As the AXIS camera is a dual-headed camera both detector heads are present in the simulations so that backscattering from one head onto the other can be modelled, which is important for the sensitivity, spectra, scatter profiles and for resolution analysis. Various source distributions are possible, including patient data. The software extension to introduce voxelized, inhomogeneous and anthropomorphic phantoms as an attenuation or as an emission map in the simulations is described in section 2.1.3 and illustrated in figure 4 for real patient data and for a digital phantom while results on this are obtained in section 3.1.

In section 3.2 we presented the comparison of energy spectra in air and with an attenuation material. The energy resolution was modelled with an energy-dependent blurrer. Figure 10(a) shows good agreement for a ${ }^{99 \mathrm{~m}} \mathrm{Tc}$ energy spectrum in air. It can be concluded from the general shape alignment that the camera is well modelled and that scatter processes in the detector are accurately simulated. Additionally the good alignment of the $70-80 \mathrm{keV}$ peak indicates that the appropriate amount of lead $\mathrm{x}$-rays are simulated. Figure 10(b) shows similar agreement for the energy resolution if a scattering and attenuating medium is present in the setup, indicating the accurate simulation of phantom scatter. Similarly, figure 11 showed good agreement for three other isotopes in air which covered a large energy range. The scintillation process and optical photon transport were not modelled for SPECT because there is only one crystal block, so that the scintillation photons are averaged over a high number of PMTs. Moreover, with a LEHR or MEGP collimator, all incidences are nearly perpendicular. The behaviour of the energy blurrer for the energy resolution satisfies in this way most needs of SPECT studies.

In section 3.3 we discussed the results of the sensitivity experiments. We compared the resulting absolute sensitivity of the simulator (LEHR collimator, no energy windows) with a realistic acquisition and a deviation between experiment and simulation of only $6.1 \%$ was found. This discrepancy was probably due to the lack of knowledge on the behaviour of the camera below $40 \mathrm{keV}$ in the measurement. Additionally, we evaluated the sensitivity in two distinct energy windows, for both collimators: one window in the photopeak region, the other in the Compton region. We concluded that the simulated values were registered within the error bars of the measured values, ruling out the possibility of inter-window compensating errors as shown in figure 12. Sensitivity results for both the photopeak window and for the Compton window appeared to be very precise, which supports the analysis made for the $6.1 \%$ 
absolute sensitivity discrepancy. Furthermore the sensitivity stayed constant with distance, as is analytically proved for parallel hole collimators. Applying one of GATE's dead time models in the simulations was thus redundant in all these low count studies.

The sensitivity result on both collimators for the Compton energy window was also a first indication of a correct modelling of the scatter processes in the detector and in the collimator. This topic was further investigated in section 3.4. Scatter profiles of a point source in air for both the photopeak window (figure 13) and for the Compton window were simulated. In the latter case a comparison with measurements was made and good agreement was reached which proves that the scatter contribution from the collimator and from the detector is correctly simulated (figure 14). To evaluate the simulator in an attenuating and scattering medium, we simulated scatter profiles of a line source in water at different depths (figure 15). Precise results were achieved after comparison with real measurements, which indicates a good scatter process simulation.

The quality of the collimator model was investigated in more detail in section 3.5 for both the LEHR and for the MEGP. The FWHM of the simulated and acquired images of line sources in air appears to be equal within the error margins as figure 16 demonstrates.

For the Beacon device a scatter analysis (figures 17(a) and (b)) was performed. It became clear that the high energy ${ }^{133} \mathrm{Ba}$ photons interacted to a large extent before reaching the detector. Multiple scattering in the collimator is evident as the ${ }^{133} \mathrm{Ba}$ source forms a transmission image through the collimator of the opposite detector. This led to the interpretation of the energy spectrum for simultaneous emission and transmission imaging. We concluded that the ${ }^{133} \mathrm{Ba}$ attenuation source contaminates the attenuation energy window to a large extent after interacting in the collimator which can possibly degrade the transmission image quality. The data of figure 17(b) for the emission peak also show that backscattering on the PMTs and the light guide largely influences the result as the high energy photons easily cross the $0.95 \mathrm{~cm}$ thin crystal. The ${ }^{133} \mathrm{Ba}$ photons lose a vast amount of energy due to this backscattering and contaminate in this way the emission window of the ${ }^{99 \mathrm{~m}} \mathrm{Tc}$ source in the case of simultaneous emission and transmission imaging which is confirmed in figure 17(c). Further research will be performed towards an appropriate correction technique.

In the Solstice case study we compared simulated sensitivity profiles with published values and a good match was achieved in figure 18(c). This proves that GATE is flexible enough to model prototype camera designs and can be used in studies where experimental setups are not available. Further validation and application modelling for Solstice are currently under investigation.

\section{Conclusion}

In this paper we have discussed the software extensions that were implemented to make GATE an accurate simulation tool for SPECT imaging. We have therefore modelled all aspects of a scintillation camera, including different collimators. An image reader for digital clinical data was also integrated into the simulation platform, making it possible to incorporate voxelized inhomogeneous phantoms in the simulations. After discussing the possibilities of GATE, the most important simulator aspects for SPECT imaging were validated: energy spectra of different isotopes, sensitivity, scatter profiles and spatial resolution. The results of this validation process were accurate and promising for future applications. Further research on variance reduction has to be accomplished to decrease the computation time. Bootstrapping and jackknifing will also be investigated in that context. Other topics of further research are scintillation modelling and light photon transport incorporation. 
We believe we have demonstrated that GATE needs very few assumptions when describing real camera designs, making it in that way very flexible. Especially the fact that no essential parts of the camera needed to be represented by a simplifying model led to the possibility of using GATE for simulating existing camera setups in different acquisition modes with different parameters, different isotopes and different collimators. Moreover this also led to the fact that complicated devices such as the Beacon attenuation correction tool and the future Solstice detector could be modelled and that accurate results could be achieved on both of them. GATE can be applied in correction technique examination, in protocol optimization and especially in future camera design.

\section{Acknowledgments}

This work was supported by the Institute for the Promotion of Innovation by Science and Technology in Flanders (IWT, Belgium), the Fund for Scientific Research Flanders (FWO, Belgium), Ghent University and by the Swiss National Foundation for Research under grant no 21-63870.00. We wish to thank Professor Christian Morel of the Institute of High Energy Physics of the University of Lausanne, Switzerland for initiating the development of GATE in Lausanne and for numerous helpful discussions. We sincerely appreciate the improvements suggested by Irene Buvat, Unity 494 INSERM, Paris, France and by the other reviewers of this manuscript. We also want to thank Professor R A Dierckx of the Department of Nuclear Medicine, Ghent University Hospital, Belgium for his helpful comments on the experiments that were described above.

\section{References}

Agostinelli S et al 2002 Technical Report SLAC-PUB-9350

Andreo P 1991 Monte Carlo Techniques in medical radiation physics Phys. Med. Biol. 36 861-920

Anger H 1964 Gamma ray efficiency and image resolution in sodium iodide Rev. Sci. Instrum. 35 693-97

Briesmeister J F et al 1988 Technical Report LA-12625-M

Brun R and Rademakers F 1997 ROOT-an object oriented data analysis framework Nucl. Instrum. Methods Res. A 389 81-6

Buvat I and Castiglioni I 2002 Monte Carlo simulations in SPET and PET Q. J. Nucl. Med. $4648-61$

Du Y, Frey E, Wang W, Tocharoenchai C, Baird W and Tsui B 2002 Combination of MCNP and SimSET for Monte Carlo simulation of SPECT with medium- and high-energy photons IEEE Trans. Nucl. Sci. 49 668-74

Gagnon D, Zeng G, Links M, Griesmer J and Valentino G 2001 Proc. IEEE Med. Im (San Diego) vol 2 pp 1156-60

GATE 2001 GATE website: http://www-iphe.unil.ch/PET/research/gate/

Griesmer J, Kline B, Grosholz J, Parnham K and Gagnon D 2001 Proc. IEEE Med. Im (San Diego) vol 2 pp 1050-4

Harrison R, Vannoy S, Haynor D, Gillispie S, Kaplan M and Lewellen T 1993 A public-domain simulation system for emission tomography: photon tracking through heterogeneous attenuation using importance sampling $\mathrm{J}$. Nucl. Med. 34 60P

Interfile 1993 Interfile website: http://gamma.wustl.edu/interfile33

Ljungberg M and Strand S E 1989 A Monte Carlo program for the simulation of scintillation camera characteristics Comput. Methods Prog. Biomed. 29 257-72

Ljungberg M, Strand S E and King M A 1998 Monte Carlo Calculations in Nuclear Medicine: Applications in Diagnostic Imaging (Bristol: Institute of Physics Publishing)

Nelson W R et al 1985 Technical Report SLAC-265

Philips, The AXIS scintillation camera is manufactured by Philips Medical Systems, 595 Miner Road, Cleveland, $\mathrm{OH} 44143$, USA

Santin G, Strul D, Lazaro D, Simon L, Krieguer M, Vieira Martins M, Breton V and Morel C 2002 GATE, a Geant4-based simulation platform for PET integrating movement and time management IEEE Trans. Nucl. Sci. at press

Sorenson J A and Phelps M E 1987 Physics in Nuclear Medicine (Philadelphia, PA: Saunders) 
Thomson C J, Cantu J M and Picard Y 1992 PETSIM: Monte Carlo program simulation of all sensitivity and resolution parameters of cylindrical positron imaging systems Phys. Med. Biol. 37 731-49

Yanch J C, Dobrzeniecki A B, Ramanathan C and Behrman R 1992 Physically realistic Monte Carlo simulation of source, collimator and tomographic data acquisition for emission computed tomography Phys. Med. Biol. 37 $853-70$

Zaidi H 1999 Relevance of accurate Monte Carlo modelling in nuclear medical imaging Med. Phys. 26 574-608

Zaidi H, Hermann Scheurer A K and Morel C 1999 An object-oriented Monte-Carlo simulator for 3D cylindrical positron tomographs Comput. Methods Prog. Biomed. 58 133-45

Zaidi H and Sgouros G 2002 Therapeutic Applications of Monte Carlo Calculations in Nuclear Medicine (Bristol: Institute of Physics Publishing)

Zeng L, Gagnon D, Matthews C, Kolthammer J, Radachy J and Hawkins W 2001a Proc. IEEE Med. Im. (San Diego) vol 4 pp 1869-73

Zeng L, Gullberg G and Christian P 2001b Asymmetric cone-beam transmission tomography IEEE Trans. Nucl. Sci. 48 117-24 